\title{
Les étudiants de la faculté de médecine dentaire au Maroc sont-ils motivés?
}

\author{
Are Moroccan dental medicine students motivated?
}

\author{
Bouchra BADRE ${ }^{1}$, Zineb SERHIER ${ }^{2}$, Mohammed BENNANI-OTHMANI ${ }^{2}$, \\ Samira EL ARABI ${ }^{1}$ \\ 1 Service de pédodontie-prévention, Faculté de médecine dentaire de Casablanca, Maroc \\ 2 Laboratoire d'Informatique médicale, Faculté de médecine et de pharmacie de Casablanca, Maroc
}

Manuscrit reçu le 8 novembre 2012 ; commentaires éditoriaux formulés aux auteurs le 9 mars 2013 ; accepté pour publication le 16 octobre 2016

\begin{abstract}
Mots-clés
Motivation ;

étudiant ; dentaire

Keywords

motivation ; dental

students

Résumé - Contexte : La motivation est une composante importante de l'apprentissage et de la performance. Il est important de la quantifier et de mesurer son évolution dans un parcours de formation long. Peu d'études ont été réalisées dans le domaine de la médecine dentaire. But : Mesurer et comparer le degré de motivation des étudiants de la faculté de médecine dentaire au début et en fin de formation. Méthodes : Étude transversale réalisée en 2011 à la faculté de médecine dentaire de Casablanca. Un questionnaire anonyme, comportant les variables sociodémographiques et une échelle pour mesurer le degré de motivation (SMMS), a été distribué à l'ensemble des étudiants de première et cinquième années. Le calcul des scores était réalisé par la somme des réponses aux items. Le score minimum est de 15 correspondants à l'absence de motivation et le score maximum est de 75 correspondants à une grande motivation. Résultats : Au total, 105 et 85 étudiants en $1^{\text {ère }}$ et $5^{\text {ème }}$ année respectivement avaient participé à l'étude. La fiabilité interne de l'échelle était bonne $(\alpha=0,75)$. Les étudiants étaient modérément motivés avec un score SMMS de 48, 1 avec $\mathrm{IC}_{95}$ (46,9-49,3). Les étudiants de première année étaient significativement plus motivés que ceux de la cinquième année (moyenne du score SMMS de 51,2 (ET = 7,3) versus 44,1 $\left.(\mathrm{ET}=7,81), p<10^{-4}\right)$. Après stratification sur l'année d'étude, les étudiantes de sexe féminin étaient plus motivées que les garçons en première année. Conclusion : Le degré de motivation des étudiants en fin de formation était plus faible que celui des étudiants en première année, d'où la nécessité de renforcer la motivation de ces étudiants.

Abstract - Background: Motivation is an important component of learning and performance. It is important to quantify and measure its progress during training. Little research has been conducted about this subject in the field of dentistry. Aim: The objective of this study was to measure and compare the level of motivation among faculty-of-dentistry students at the beginning and the end of training. Methods: A cross-sectional study was conducted in
\end{abstract}


2011 at the faculty of dentistry of Casablanca. An anonymous questionnaire including sociodemographic variables and a scale to measure the degree of motivation (SMMS) was distributed to all students in the first and fifth years. The scoring was carried out by adding up the responses. The minimum score was 15 , consistent with a lack of motivation and the maximum score was 75, consistent with very high motivation. Results: A total of 105 and 85 students in the first and fifth years respectively participated in the study. The internal reliability of the scale was good $(\alpha=0.75)$. Students were moderately motivated with a SMMS score of 48.1 (95\% CI: 46.9 to 49.3 ). First-year students were significantly more motivated than fifthyear students. The SMMS average score was $51.2(\mathrm{SD}=7.3)$ versus $44.1(\mathrm{SD}=7.81), p<$ $10^{-4}$. Female students were more motivated than males in first year. Conclusion: Students' motivation at the end of training was significantly lower than that of first-year students, hence the need to support and enhance student motivation during training.

\section{Introduction}

La motivation en milieu scolaire est définie par Viau comme un état dynamique qui a ses origines dans les perceptions qu'un élève a de lui-même et de son environnement, qui l'incite à choisir une activité, à s'y engager et à persévérer dans son accomplissement afin d'atteindre un but ${ }^{[1]}$.

Il existe différents aspects de la motivation ; on peut distinguer respectivement la qualité de la motivation ou les facteurs motivationnels, ces derniers pouvant être intrinsèques ou extrinsèques, et le degré de motivation qui en mesure la force indépendamment des raisons derrière le choix ${ }^{[2]}$.

Dans les études universitaires, la motivation est associée à l'orientation vers un domaine d'études précis, à l'engagement dans l'effort d'apprendre, à la discipline qu'on s'impose pour atteindre ses buts, à la persévérance face aux difficultés et aux échecs qu'on rencontre ${ }^{[3]}$. On peut croire que les étudiants à l'université sont forcément motivés, étant donné qu'à ce niveau ils font le choix de leurs études. Cependant, les enseignants constatent parfois un certain pourcentage d'absentéisme aux cours et aux travaux dirigés, ainsi qu'un investissement insuffisant dans les stages cliniques, jugé par le nombre faible d'actes réalisés durant les vacations cliniques. Cette faible implication pourrait être en relation avec une faible motivation.

Selon Viau et al. ${ }^{[4]}$, la faible motivation à apprendre peut caractériser certains étudiants universitaires de premier cycle et constitue une des raisons principales qui les amènent à abandonner leurs études. Par ailleurs, des résultats d'enquêtes ont montré que plus les étudiants avancent dans leurs études, moins ils se sentent motivés et plus leur intérêt et leur curiosité scientifique ainsi que leur persévérance diminuent ${ }^{[5,6]}$. Cette diminution de motivation peut retentir sur la performance des étudiants ${ }^{[1]}$.

Plusieurs études, dans différents pays, se sont intéressées à la motivation chez les étudiants en médecine et en médecine dentaire. La plupart des études se sont intéressé aux facteurs liés au choix de la carrière médicale. Les études qu'on a pu retrouver ayant porté sur la mesure du degré de motivation avaient utilisé le questionnaire «Strength of Motivation for Medical School » (SMMS) ${ }^{[2,7-9]}$.

Au Maroc, l'accès à la faculté de médecine dentaire est ouvert aux élèves titulaires d'un baccalauréat des séries sciences expérimentales, sciences mathématiques ou sciences agronomiques, après une présélection suivi d'un concours. La formation en médecine dentaire dure cinq ans après lesquelles l'étudiant doit soutenir une thèse. Le nombre réduit de facultés de médecine dentaire qui sont au nombre de deux, une à Casablanca et une à Rabat, explique le caractère hautement sélectif des études en médecine dentaire. Ainsi, l'admission à cette formation nécessite un investissement et une préparation considérables de la part des étudiants au cours des études secondaires, afin de constituer un dossier académique de haut niveau. Ces efforts fournis par les étudiants pour intégrer la formation en médecine dentaire laissent supposer qu'ils sont motivés. Cependant, à notre 
connaissance aucune étude n'a été publiée sur le degré de motivation chez les étudiants en médecine dentaire. La connaissance du niveau de motivation des étudiants et la mise en évidence d'une éventuelle diminution au cours du cursus de formation montrerait l'intérêt de la mise en place de nouvelles stratégies afin de remédier au problème de démotivation.

L'objectif de cette étude était de mesurer et de comparer le degré de motivation chez les étudiants de la faculté de médecine dentaire au début et en fin de formation.

\section{Méthodes}

Il s'agit d'une étude transversale réalisée en 2011 à la Faculté de médecine dentaire de Casablanca.

Un questionnaire auto-administré anonyme a été distribué aux étudiants de la première et de la cinquième année de la faculté de médecine dentaire vers la fin du deuxième semestre. Le questionnaire a été remis à tous les étudiants de la première année au cours des travaux dirigés lors de deux séances programmées durant la même demi-journée, regroupant l'ensemble de la promotion répartie en deux groupes. D'autres questionnaires ont été distribués une semaine après pour les étudiants qui étaient absents. Concernant les étudiants de la cinquième année, le questionnaire a été distribué à la fin de leur vacation clinique. La récupération des questionnaires a été faite immédiatement après leur remplissage. Les caractéristiques des participants sont présentées dans le tableau I.

Ce questionnaire comportait des donnés sociodémographiques et une mesure du degré de motivation par l'échelle $\mathrm{SMMS}^{[2]}$. Cette échelle explore la préparation des élèves pour commencer ou continuer leur formation médicale indépendamment de sacrifices (temps, argent, énergie). Elle comporte 16 items, dont un a été éliminé, car il n'était pas adapté au contexte marocain ; cet item porte en effet sur l'accumulation de crédits pour financer les études, alors que les étudiants marocains n'ont pas recours au crédit car les études supérieures sont financées en totalité par l'état.
Tableau I. Caractéristiques des participants.

\begin{tabular}{|l|c|}
\hline & Effectif $(\%)$ \\
\hline Année d'étude & $105(55,3)$ \\
$1^{\text {ère }}$ année & $85(44,7)$ \\
$5^{\text {ème }}$ année & $145(76,3)$ \\
\hline Sexe & $45(23,7)$ \\
Féminin & $20,5(2,3)$ \\
Masculin & \\
\hline Age moyenne (écart-type) & \\
\hline
\end{tabular}

Les items du SMMS sont cotés de 1 à 5 allant de désaccord total à accord total. Le score est calculé par la somme des réponses aux items, en inversant les réponses des items négatifs. Ce score va de 15 à 75. Les scores ont été normalisés pour varier de 0 à 100 pour faciliter leur interprétation. Un score élevé correspond à une grande motivation.

La traduction du questionnaire a été faite selon les recommandations de Beaton et al. ${ }^{[10]}$; le questionnaire original a été traduit séparément par deux traducteurs maitrisant les deux langues anglaise et française, une synthèse des versions traduites a été faite lors d'une réunion de concertation. La version française obtenue a été contre-traduite en anglais par deux autres traducteurs. La version française et les versions contre-traduites ont été comparées à la version originale, lors d'une réunion rassemblant les traducteurs, des enseignants et des médecins dentistes, afin de vérifier les équivalences sémantiques et conceptuelles.

La fiabilité interne de l'échelle a été vérifiée par le calcul du coefficient $\alpha$ de Crombach. Une description des scores a été réalisée, ainsi qu'une comparaison de ces scores selon le sexe et l'année d'étude par le test de Wilcoxon. Les données ont été analysées par le logiciel SPPSS 16.0.

\section{Résultats}

Notre étude a porté sur l'ensemble des étudiants inscrits en première et en cinquième années durant l'année universitaire 2010-2011. Le questionnaire a été acheminé à 250 étudiants ; 191 questionnaires ont 
été rendus soit $76 \%$ de réponses $(72 \%$ des étudiants de première année et $82 \%$ des étudiants de cinquième année).

La moyenne d'âge des répondants était de 20,5 \pm 2,3 ans. Les filles $(n=145)$ représentaient $76,3 \%$ des étudiants. $0,75)$.

La fiabilité interne de l'échelle était bonne ( $\alpha=$

Les étudiants étaient modérément motivés avec une médiane du score normalisé du SMMS de 56,7, un minimum de 15,0 et un maximum de 91,7.

Plus de la moitié étaient d'accord pour sacrifier leur vie sociale $(56,8 \%)$ et pour annuler les vacances avec les amis $(52,9 \%)$ pour la médecine dentaire. Environ 43,7\% avaient déclaré qu'ils choisiraient à nouveau comme formation la médecine dentaire et 43,1\% étaient d'accord pour le choix de la médecine dentaire même s'il faut étudier beaucoup de matières. Cependant $42,4 \%$ rapportaient qu'ils auraient quitté la médecine dentaire s'ils leur faillait 10 ans pour avoir le doctorat (tableau II).

En comparant les scores SMMS, les étudiants de première année étaient significativement plus motivés que ceux de la cinquième année avec une médiane de score normalisé de 61,7 versus 48,3 ( $p<$ $10^{-3}$ ) (figure 1).

Il n'y avait pas de différence significative entre le degré de motivation des filles comparativement aux garçons, avec une médiane de score normalisé de 55,0 pour les filles et de 56,7 pour les garçons ( $p=$ 0,908 ) (figure 2). Après stratification sur l'année d'étude, les étudiantes de sexe féminin étaient plus motivées que les garçons en première année (tableau II).

\section{Discussion}

Notre étude a montré une prédominance du sexe féminin chez les étudiants en médecine dentaire. Le degré de motivation de ces étudiants était modéré (score de 55,2 sur une échelle de 100). Les étudiants de première année étaient nettement plus motivés que ceux de la cinquième année et le genre féminin était associé au degré de motivation chez les étudiants de première année.
Cette étude présente quelques limites, notamment le problème des non réponses (taux de réponses $76 \%$ ). Les non-répondants pourraient présenter un profil motivationnel particulier, le refus de remplir les questionnaires pouvant être lié à leur démotivation. Ce biais de sélection pourrait être à l'origine d'une surestimation des scores de motivation. La validité de l'outil de mesure utilisé n'a pas été vérifié dans sa traduction française, cependant il a démontré une bonne fiabilité interne $(\alpha=0,75)$. Par ailleurs, dans notre étude, nous avons pris deux échantillons différents, formés par des étudiants de la première année et de la cinquième année ; pour vérifier s'il y a une réelle démotivation, il serait souhaitable de suivre un même échantillon de la première année jusqu'à la cinquième année pour décrire l'évolution de leur degré de motivation le long de leurs années d'étude.

Généralement, les étudiants choisissent la médecine dentaire, parce qu'ils veulent être indépendants, servir et aider les autres, acquérir un statut social, une sécurité financière et la flexibilité des horaires ${ }^{[11-13]}$. Ces raisons n'ont pas la même priorité pour les deux sexes ${ }^{[4]}$. Dans une étude menée sur des étudiants nigériens $^{[14]}$, environ $50 \%$ faisaient le choix d'une formation en médecine dentaire en étant influencé par la famille. D'autres facteurs interviennent aussi dans le choix de cette carrière : heures de travail régulières, prestige et bonne opportunité de travail à l'étranger. Dans une étude menée par Al bitar ${ }^{[15]}$, il a été trouvé que la sécurité professionnelle et financière était prépondérante pour les premières années dans le choix d'une carrière en chirurgie dentaire, contrairement aux cinquièmes années. Cette différence peut s'expliquer par une conception plus réaliste de cette carrière, acquise par un gain de connaissances concernant les aspects pratiques des opportunités d'emplois et le stress du métier. Ceci peut expliquer la différence de motivation observée entre les étudiants de la première et la cinquième année dans notre étude. Des résultats similaires ont été trouvés chez des étudiants québécois : une étude a montré que plus ils avancent dans leurs études, moins ils se sentent motivés ${ }^{[4]}$. La baisse de motivation entre le début et la fin de formation peut s'expliquer aussi par le degré de concordance entre 
I abıeau II. vegre de motivatıon des etudiants.

\begin{tabular}{|c|c|c|c|c|c|}
\hline & $\begin{array}{c}\text { Désaccord } \\
\text { Total }(\%)\end{array}$ & $\begin{array}{l}\text { Désaccord } \\
\quad(\%)\end{array}$ & $\begin{array}{l}\text { Sans opinion } \\
(\%)\end{array}$ & $\begin{array}{l}\text { Accord } \\
(\%)\end{array}$ & $\begin{array}{c}\text { Accord } \\
\text { Total }(\%)\end{array}$ \\
\hline Regret si pas admis en médecine dentaire & $32(16,8)$ & $41(21,6)$ & $41(21,6)$ & $46(24,2)$ & $30(15,8)$ \\
\hline $\begin{array}{l}\text { Quitter les études de médecine dentaire, si } \\
\text { impossibilité de devenir spécialiste }\end{array}$ & $32(16,9)$ & $62(32,8)$ & $41(21,7)$ & $37(19,6)$ & $17(9,0)$ \\
\hline $\begin{array}{l}\text { Choisir médecine dentaire, même dans } \\
\text { une autre langue }\end{array}$ & $53(27,7)$ & $79(41,4)$ & $23(12,0)$ & $28(14,7)$ & $8(4,2)$ \\
\hline $\begin{array}{l}\text { Quitter la médecine dentaire, s'il faut } \\
10 \text { ans pour avoir le doctorat }\end{array}$ & $24(12,6)$ & $52(27,2)$ & $34(17,8)$ & $51(26,7)$ & $30(15,7)$ \\
\hline $\begin{array}{l}\text { Sacrifier la vie sociale pour la formation } \\
\text { en Médecine dentaire }\end{array}$ & $16(8,4)$ & $30(15,8)$ & $36(18,9)$ & $80(42,1)$ & $28(14,7)$ \\
\hline $\begin{array}{l}\text { Choisir toujours comme formation la } \\
\text { Médecine dentaire }\end{array}$ & $29(15,3)$ & $39(20,5)$ & $39(20,5)$ & $54(28,4)$ & $29(15,3)$ \\
\hline $\begin{array}{l}\text { Annuler les vacances avec les amis pour } \\
\text { la médecine dentaire }\end{array}$ & $18(9,4)$ & $28(14,7)$ & $44(23,0)$ & $83(43,5)$ & $18(9,4)$ \\
\hline $\begin{array}{l}\text { Arrêter la médecine dentaire en cas de } \\
\text { mauvaise notes et échec aux examens* }\end{array}$ & $51(26,7)$ & $78(40,8)$ & $36(18,8)$ & $24(12,6)$ & $2(1,0)$ \\
\hline $\begin{array}{l}\text { Quitter la médecine dentaire, s'il faut plus } \\
\text { de } 60 \text { heures par semaine pour étudier* }\end{array}$ & $52(27,2)$ & $83(43,5)$ & $33(17,3)$ & $12(6,3)$ & $11(5,8)$ \\
\hline $\begin{array}{l}\text { Changer facilement de carrière en cas de } \\
\text { difficultés* }\end{array}$ & $80(41,9)$ & $72(37,7)$ & $22(11,5)$ & $12(6,3)$ & $5(2,6)$ \\
\hline $\begin{array}{l}\text { Persister dans les études de médecine } \\
\text { dentaire, même au dépend de la famille }\end{array}$ & $15(8,1)$ & $33(17,7)$ & $52(28,0)$ & $67(36,0)$ & $19(10,2)$ \\
\hline $\begin{array}{l}\text { Quitter la médecine dentaire, en cas de } \\
\text { problèmes de recrutement* }\end{array}$ & $41(21,5)$ & $88(46,1)$ & $30(15,7)$ & $22(11,5)$ & $10(5,2)$ \\
\hline $\begin{array}{l}\text { Ne pas choisir la médecine dentaire, en } \\
\text { cas d'impossibilité à financer les études* }\end{array}$ & $19(9,9)$ & $58(30,4)$ & $42(22,0)$ & $50(26,2)$ & $22(11,5)$ \\
\hline $\begin{array}{l}\text { Choisir la médecine dentaire, même s'il } \\
\text { faut étudier beaucoup de matières }\end{array}$ & $30(15,8)$ & $41(21,6)$ & $37(19,5)$ & $58(30,5)$ & $24(12,6)$ \\
\hline $\begin{array}{l}\text { Refaire le bac, pour avoir une meilleure } \\
\text { note pour être sélectionne pour les études } \\
\text { en médecine dentaire (pour garantir la } \\
\text { sélection en médecine dentaire) }\end{array}$ & $60(31,6)$ & $56(29,5)$ & $24(12,6)$ & $33(17,4)$ & $17(8,9)$ \\
\hline
\end{tabular}

\footnotetext{
* items négatifs.
}

Tableau III. Comparaison du degré de motivation selon le sexe en stratifiant sur l'année d'étude (score normalisé au test Measuring Strength of Motivation for Medical School) ${ }^{[2]}$.

\begin{tabular}{|l|c|c|c|c|}
\hline & $1^{\text {ère }}$ année & $p$ & $5^{\text {ème }}$ année & $p$ \\
\hline Masculin & 58,3 & 0,151 & 50,0 & \multirow{2}{*}{0,328} \\
\hline Féminin & 61,7 & & 48,3 & \\
\hline
\end{tabular}




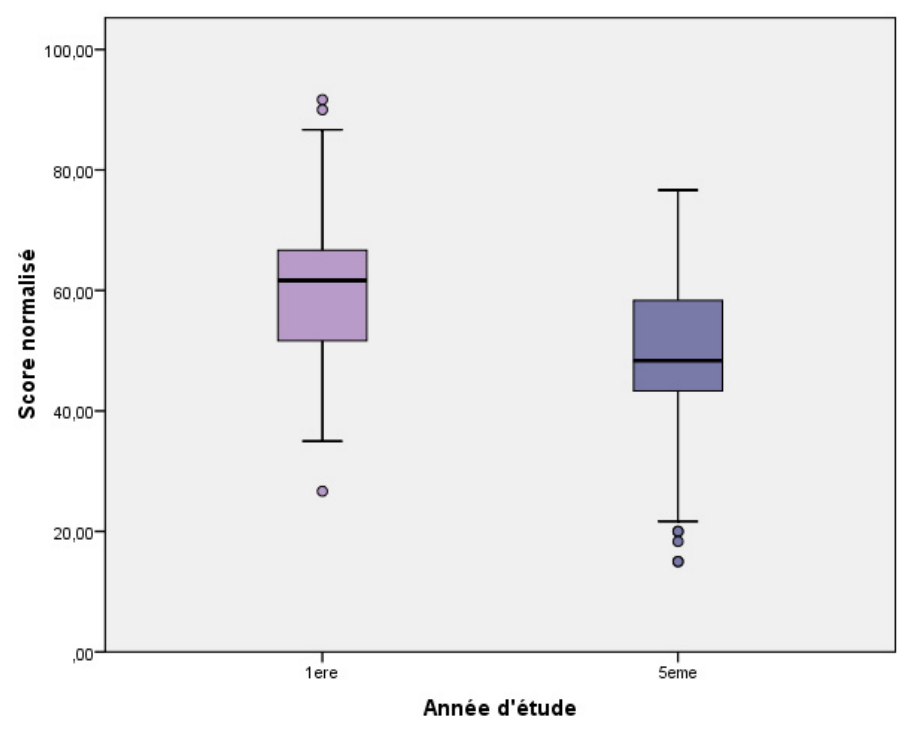

Fig. 1. Degré de motivation selon l'année d'étude. (Score normalisé au test Measuring Strength of Motivation for Medical School ${ }^{[2]}$ )

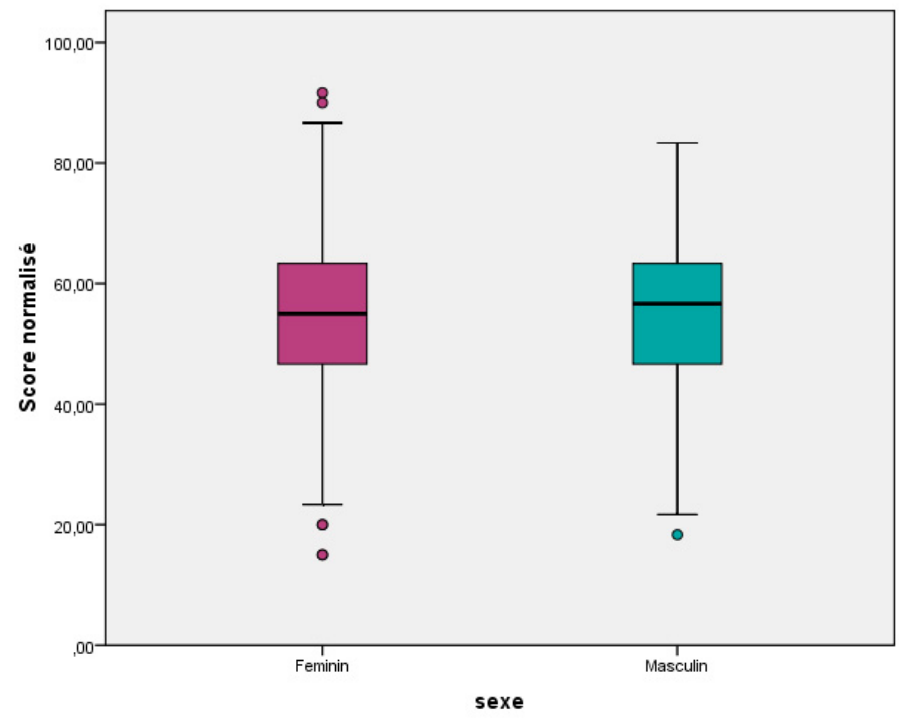

Fig. 2. Description des degrés de motivation selon le sexe. (Score normalisé au test Measuring Strength of Motivation for Medical School ${ }^{[2]}$ )

les attentes et les performances acquises ${ }^{[16]}$. Par ailleurs, selon le modèle théorique de la dynamique motivationnelle de Viau ${ }^{[17]}$, il ne s'agit pas d'un phénomène stable dans le temps mais d'un phénomène évolutif qui est déterminé par la perception de la tâche et le contexte dans lequel l'activité est réalisée.
Ainsi plusieurs facteurs sont susceptibles d'influencer la motivation des étudiants. En plus des facteurs relatifs à la vie personnelle de l'individu (notamment, l'influence familiale) et à la société et ses valeurs, il existe également de nombreux facteurs liés à l'environnement d'apprentissage. Parmi ces 
derniers, on trouve en particulier les règles de fonctionnement de l'institution, les activités pédagogiques et les pratiques évaluatives. Viau considère ainsi que ces facteurs jouent un rôle prépondérant sur la motivation. Au niveau de la faculté de médecine dentaire de Casablanca, depuis quelques années, des séances d'apprentissage au raisonnement clinique et des dispositifs répondant au format de l'apprentissage par problèmes ont été introduits dans le cursus de formation afin de favoriser chez les étudiants l'acquisition d'une autonomie vis-à-vis de leurs apprentissages. Cette autonomie est indispensable pour l'acquisition de nouvelles connaissances et de compétences, en permettant ainsi une amélioration probable de la perception qu'a l'étudiant de sa compétence, qui constitue l'un des déterminants de la motivation. D'autres mesures sont à mettre en place afin d'éviter la diminution de la motivation au cours de la formation ; concernant les activités pédagogiques, il est important de rendre explicite les liens entre les connaissances théoriques et pratiques et de montrer la pertinence du cours dans le cursus et par rapport aux besoins professionnels. En effet, Barker et Olson ${ }^{[18]}$ avaient rapporté que la diminution de la motivation intrinsèque des étudiants de première année de médecine peut être en rapport avec le fait qu'ils ont du mal à trouver la pertinence de ce qu'ils font durant cette année d'étude par rapports à leur projet d'être médecin praticien. Au niveau des stages cliniques, l'étudiant doit avoir plus d'autonomie dans la prise en charge des patients qui lui sont confiés. Pour l'évaluation en stage, notre faculté a adopté, depuis cinq ans, un outil d'évaluation en ligne qui permet aux étudiants de suivre leur évolution en matière de stages cliniques en consultant les appréciations des enseignants concernant chaque acte réalisé. Cependant, on note l'absence de l'évaluation formative car tout acte réalisé est comptabilisé pour la validation des stages. Pour remédier à cette situation, il est nécessaire de consacrer un certain nombre de vacations à la réalisation d'actes qui ne seront pas pris en considération dans la note finale.

La réussite des étudiants à l'université dépend d'une multitude de facteurs, parmi lesquels leur motivation à apprendre. L'amélioration des conditions d'apprentissage et d'évaluation est essentielle au maintien de la motivation des étudiants en formation car ce sont les leviers d'action sur lesquels il sera possible d'agir, contrairement aux facteurs relatifs à l'environnement familial et social de l'étudiant.

\section{Contributions}

Bouchra Badre a élaboré le protocole de recherche et rédigé la première version du manuscrit. Les autres auteurs ont participé à la collecte et à l'interprétation des données et ont contribué à la révision du manuscrit.

\section{Déclaration d'intérêt}

Les auteurs déclarent ne pas avoir de conflits d'intérêts en lien avec le contenu de cet article

\section{Approbation éthique}

Non sollicitée

\section{Références}

1. Viau R. La motivation en contexte scolaire. Bruxelles : De Boeck, 2005.

2. Nieuwhof MG, ten Cate OT, Oosterveld P, Soethout MB. Measuring Strength of Motivation for Medical School. Med Educ Online 2004;9:16-22.

3. Careau L, Fournier A-L. La motivation. Université Laval. 2002 [On-line]. Disponible sur : http://bibliotheque.cegeptr.qc.ca/public/8fb3d2ee-8c32-43c49851-2df6895b1ccc/documents/ 7_guide_motivation.pdf

4. Viau R, Joly J, Bédard D. La motivation des étudiants en formation des maîtres à l'égard d'activités pédagogiques innovatrices. Revue des sciences de l'éducation 2004;30:163-76. 
5. Bédard D, Viau R. Le profil d'apprentissage des étudiantes et des étudiants de l'Université de Sherbrooke: résultats de l'enquête menée au trimestre d'automne 2000. Sherbrooke (QC) : Université de Sherbrooke, 2001.

6. Pelaccia T, Delplancq H, Triby E, Leman C, Bartier J-C, Dupeyron J-P. La motivation en formation: une dimension réhabilitée dans un environnement d'apprentissage en mutation. Pédagogie Médicale 2008;9:103-21.

7. Kusurkar R, Kruitwagen C, ten Cate O, Croiset G. Effects of age, gender and educational background on strength of motivation for medical school. Adv Health Sci Educ Theory Pract 2010;15:303-13.

8. Kusurkar R, Croiset G, Kruitwagen C, Cate O. Validity evidence for the measurement of the strength of motivation for medical school. Adv Health Sci Educ Theory Pract 2010;16:183-95.

9. Hulsman RL, van der Ende JSJ, Oort FJ, Michels RPJ, Casteelen G, Griffioen FMM. Effectiveness of selection in medical school admissions: evaluation of the outcomes among freshmen. Med Educ 2007;41: 369-77.

10. Beaton DE, Bombardier C, Guillemin F, Ferraz MB. Guidelines for the process of cross-cultural adaptation of self-report measures. Spine 2000;25:3186-91.

11. Lalloo R, Ayo-Yusuf OA, Yengopal V. Early-phase dental students' motivations and expectations concerning the study and profession of dentistry. SADJ 2008;63:216-20

12. Hawley NJ, Ditmyer MM, Sandoval VA. Predental students' attitudes toward and perceptions of the dental profession. J Dent Educ 2008;72:1458-64.

13. Khami MR, Murtomaa H, Jafarian M, Vehkalahti MM, Virtanen JI. Study motives and career choices of Iranian dental students. Med Princ Pract 2008; 17:221-6

14. Orenuga OO, Costa OO da. Characteristics and Study Motivation of Clinical Dental Students in Nigerian Universities. J Dent Educ 2006;70:996-1003.

15. Al-Bitar ZB, Sonbol HN, Al-Omari IK. Reasons for choosing dentistry as a career by Arab dental students. Eur J Dent Educ 2008;12:247-51.

16. Kristensen BT, Netterstrom I, Kayser L. Dental students' motivation and the context of learning. Eur J Dent Educ 2009; 13:10-4.

17. Viau R. La motivation en contexte scolaire. Bruxelles : De Boeck Supérieur 2009

18. Barker JR, Olson JP. Medical students' learning strategies: evaluation of first year changes. J Miss Acad Sci 1997;42:96-10.

Correspondance et offprints : Bouchra Badre, Service de pédodontie-prévention, Faculté de médecine dentaire de Casablanca (Maroc).

Mailto : bouchrabadre@hotmail.fr 